\title{
Allergy to Strawberry in Children From the Mediterranean Area: Is It Really Allergy?
}

Cabrera-Freitag $\mathrm{P}^{1,2}$, Bermejo Becerro $\mathrm{A}^{1}$, Abreu Ramírez $\mathrm{MG}^{1}$, Álvarez-Perea $\mathrm{A}^{1,2}$, Infante Herrero $\mathrm{S}^{1,2}$, Fuentes-Aparicio $\mathrm{V}^{1,2}$, Zapatero Remón $\mathrm{L}^{1,2}$

${ }^{1}$ Pediatric Allergy Unit, Hospital General Universitario Gregorio Marañón, Madrid, Spain

${ }^{2}$ Gregorio Marañón Health Research Institute (IiSGM), Madrid, Spain

J Investig Allergol Clin Immunol 2020; Vol. 30(4): 283-285 doi: $10.18176 /$ jiaci.0491

Key words: Strawberry allergy. Children. Rosaceae family.

Palabras clave: Alergia a fresa. Niños. Familia Rosaceae.

Members of the Rosaceae family are the most frequent cause of allergic reactions to fruits in the Mediterranean area [1]. Strawberry, which belongs to the Rosoideae subfamily of Rosaceae, has an apparently unjustified poor reputation among the general population, as self-reported symptoms after ingestion of strawberry are very common [2,3]. However, few cases of true allergy have been reported in the literature [4-7].

The aim of our study was to make a descriptive analysis of pediatric patients with a history of self-reported strawberry allergy and to investigate whether they had true allergy. Patients from the Pediatric Allergy Department of Hospital General Universitario Gregorio Marañón, Madrid, Spain were retrospectively analyzed on the basis of a clinical history of strawberry allergy, specific IgE (sIgE) to strawberry, and age under 17 years.

The data we recorded included demographic and clinical characteristics, specific IgE (sIgE) values to strawberry (ImmunoCAP 250, Thermo Fisher Scientific), skin prick test (SPT) results with a commercial strawberry extract (Leti), sensitization to profilin by prick and peach nonspecific lipid transfer protein (nsLTP) by prick (peach extract enriched with Pru p 3 [ALK-Abelló] or Pru p 3 [ImmunoCAP]), and tolerance to strawberry in oral food challenge (OFC). sIgE values to birch PR-10 (Bet v 1) were not analyzed, as sensitization to birch pollen is not common in our area. SPT wheals $\geq 3 \mathrm{~mm}$ and $\mathrm{sIgE}$ values $\geq 0.35 \mathrm{kU} / \mathrm{L}$ were considered positive.

Qualitative variables are expressed as a frequency and quantitative variables as median (IQR). Categorical variables were compared using the $\chi^{2}$ test and Fisher exact test; quantitative variables were compared using the MannWhitney test.

The study population comprised 43 children with a clinical history of strawberry allergy. Of these, $29(67 \%)$ had a positive SPT and/or sIgE result to strawberry (group 1) and 14 (33\%) had negative results in both tests (group 2).

Median time between self-reported symptoms related to strawberry intake and the allergological work-up was 4 (3-6) months; median time from symptoms to the assessment of 
tolerance was 6 (4-9) months. Cofactors such as concomitant exercise, infectious disease, and nonsteroidal antiinflammatory drug intake were excluded in all patients.

Among patients belonging to group 1 (58.6\% male, median age 9 [6-12] years), the most frequently reported symptoms were pruritus of the oral mucosa (oral allergy syndrome [OAS]) and cutaneous symptoms (48.3\% and $37.9 \%$, respectively). Three patients $(10.3 \%)$ reported gastrointestinal symptoms and 1 anaphylaxis (3.4\%). All patients also had concomitant atopic diseases: 23 patients $(79.3 \%)$ were allergic to other foods (mostly other fruits $[\mathrm{n}=20]$, with peach the most frequently involved [39.3\%] in fruit-allergic patients), $16(55.1 \%)$ had rhinoconjunctivitis and/or bronchial asthma related to aeroallergens other than birch, and 13 (44.8\%) had atopic dermatitis.

Symptoms at onset in patients belonging to group 2 (57.1\% male, median age 4.5 [2-12] years) comprised OAS $(50 \%)$ and cutaneous symptoms (50\%). All but 1 patient had at least another atopic disease: $7(50 \%)$ had atopic dermatitis, $6(42.8 \%)$ had at least 1 other food allergy (with fruits

Table. Result of the Allergological Work-up

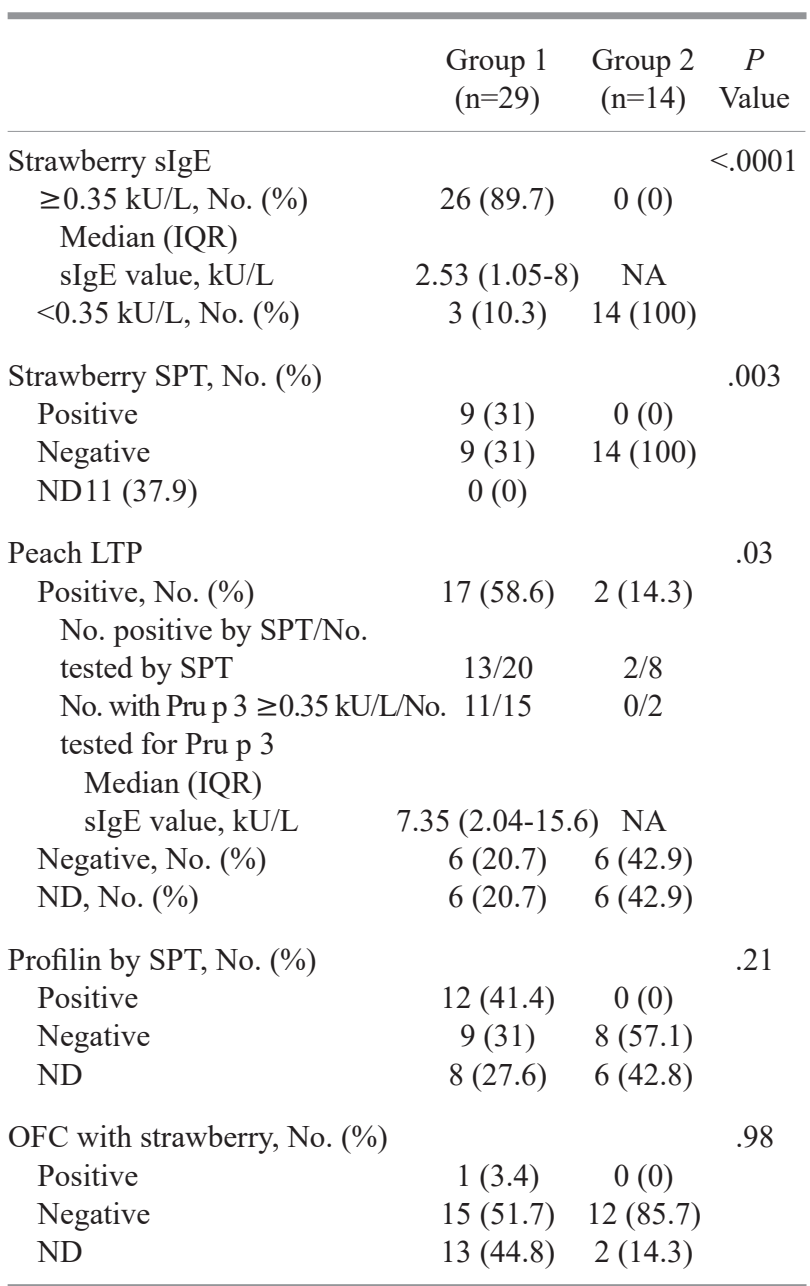

Abbreviations: NA, not applicable; ND, not done; OFC, oral food challenge; SPT, skin prick test. being the most frequently involved $[\mathrm{n}=4])$, and $5(35.7 \%)$ had rhinoconjunctivitis and/or bronchial asthma related to aeroallergens but not birch.

No statistical differences were observed regarding gender, age, or type of symptoms between groups. Patients in group 1 were more frequently allergic to other foods and fruits than those in group 2 ( $\mathrm{p}=0.03$ and 0.01 respectively), although no differences were observed for other atopic diseases.

The results of the allergological work-up are shown in the Table. Tolerance was assessed in 28 children $(65.1 \%$, 16 belonging to group 1 and 12 to group 2), with a dose proportionate to their age, and all but 1 tolerated strawberry $(96.4 \%)$. There were no significant differences between patients belonging to group 1 in whom tolerance to strawberry was assessed and those in whom it was not regarding age, clinical symptoms, concomitant atopic diseases, sIgE values to strawberry, and SPT results with strawberry, profilin, and nsLTP. These data were not analyzed for patients belonging to group 2 owing to the small sample (12/14 tested for tolerance vs $2 / 14$ not tested).

All but 1 child in group 1 (16/29 tested) tolerated strawberry $(93.7 \%)$ : 3 were not allergic to other fruits, 7 were allergic to peach, 3 to Rosaceae fruits other than peach, and 2 to fruits other than Rosaceae. The patient who did not tolerate strawberry had a clinical history of anaphylaxis with strawberry, a positive SPT and ImmunoCAP result to strawberry $(2.47 \mathrm{kU} / \mathrm{L})$, and a positive SPT to profilin (sensitization to LTP not tested). This boy was also allergic to apple, house dust mite, and plane tree pollen. All children tested in group 2 tolerated strawberry.

Consistent with other studies carried out in southern Europe, most of the patients in our study who self-reported symptoms after strawberry consumption experienced mild symptoms (OAS and cutaneous symptoms) and were allergic to other fruits, mostly peach $[4,5,7]$. Moreover, $96 \%$ of the children in our study with symptoms after strawberry intake tolerated the fruit in a subsequent OFC, thus supporting the idea that true allergy to strawberry is not as frequent as it seems. Since this was independent of whether or not they were sensitized to strawberry, neither SPT nor CAP seem to have good sensitivity, although specificity was good, as all patients with negative results in both diagnostic tests tolerated strawberry.

The high percentage of patients sensitized to peach LTP (61.3\% [19/31 tested]) and profilin (41.4\% [12/29 tested]) could partly explain the patient's sensitization to strawberry due to cross-reactivity [4].

Our study suggests that true allergy to strawberry in our part of the Mediterranean area is rare. Therefore, we believe that, in our region, OFC should be considered in children who report mild symptoms (OAS and/or cutaneous symptoms) after strawberry intake, regardless of whether or not they are sensitized to strawberry, and even in those who are sensitized to LTP. Nevertheless, in patients with more severe symptoms, true allergy to strawberry might be considered.

Further studies involving more patients are needed in order to analyze whether severity of the symptoms and strawberry allergen sensitization profile are associated with true strawberry allergy. 


\section{Funding}

The authors declare that no funding was received for the present study.

\section{Conflicts of Interest}

The authors declare that they have no conflicts of interest.

\section{References}

1. Lyons SA, Burney PGJ, Ballmer-Weber BK, Fernandez-Rivas $M$, Barreales $L$, Clausen $M$, et al. Food Allergy in Adults: Substantial Variation in Prevalence and Causative Foods Across Europe. J Allergy Clin Immunol Pract. 2019;7(6):19208.

2. Jorge A, Soares E, Sarinho E, Lorente F, Gama J, TabordaBarata L. Prevalence and clinical features of adverse food reactions in Portuguese children. Allergy Asthma Clin Immunol. 2017;13(1):40.

3. Venter C, Pereira B, Grundy J, Clayton CB, Arshad SH, Dean T. Prevalence of sensitization reported and objectively assessed food hypersensitivity amongst six-year-old children: a population-based study. Pediatr Allergy Immunol. 2006;17(5):356-63.

4. Zuidmeer L, Salentijn E, Rivas MF, Mancebo EG, Asero R, Matos $\mathrm{Cl}$, et al. The role of profilin and lipid transfer protein in strawberry allergy in the Mediterranean area. Clin Exp Allergy. 2006;36(5):666-75.

5. Rodriguez J, Crespo JF, Lopez-Rubio A, De La Cruz-Bertolo $J$, Ferrando-Vivas P, Vives R, et al. Clinical cross-reactivity among foods of the Rosaceae family. J Allergy Clin Immunol. 2000; 106(1 Pt 1):183-9.

6. Karamloo F, Wangorsch A, Kasahara $H$, Davin LB, Haustein $D$, Lewis NG, et al. Phenylcoumaran benzylic ether and isoflavonoid reductases are a new class of cross-reactive allergens in birch pollen, fruits and vegetables. Eur J Biochem. 2001;268(20):5310-20.

7. Pajno GB, Passalacqua G, La Grutta S, Vita D, Feliciotto R, Parmiani $S$, et al. True multifood allergy in a 4-year-old child: a case study. Allergol Immunopathol (Madr). 2002;30(6):33841.

Manuscript received September 25, 2019; accepted for publication January 30, 2020.

Paula Cabrera Freitag

E-mail: paula.cabrera@salud.madrid.org 